\title{
L'évolution de l'expertise médicale des guérisons de Lourdes au regard de la « conversation de gestes »
}

Introduction : de quoi rient les rationalistes?

The evolution of the medical expertise of Lourdes' cures relatively to the " conversation de gestes"

La evolución de la expertise médica de las sanaciones de Lourdes

en relación con la "conversación de gestos"

\section{Laetitia Ogorzelec}

\section{OpenEdition}

Journals

Édition électronique

URL : http://journals.openedition.org/assr/26037

DOI : $10.4000 /$ assr. 26037

ISSN : $1777-5825$

Éditeur

Éditions de l'EHESS

Édition imprimée

Date de publication : 10 juillet 2014

Pagination : 221-242

ISBN : 978-2-7132-2432-4

ISSN : 0335-5985

Référence électronique

Laetitia Ogorzelec, «L'évolution de l'expertise médicale des guérisons de Lourdes au regard de la " conversation de gestes » », Archives de sciences sociales des religions [En ligne], 166 | Avril-Juin 2014, mis en ligne le 10 juillet 2017, consulté le 19 avril 2019. URL : http://journals.openedition.org/ assr/26037 ; DOI : 10.4000/assr.26037 
Laetitia Ogorzelec

\section{L'évolution de l'expertise médicale des guérisons de Lourdes au regard de la " conversation de gestes"}

\section{Introduction : de quoi rient les rationalistes?}

"Celui qui veut tuer le plus complètement se met à rire. "

(Nietzsche, 1993 : 535)

En janvier 1910, à la Société Médicale de Milan dont il est membre, le Docteur Gemelli expose à ses confrères « les raisons scientifiques qui l'ont amené à conclure à la possibilité de guérisons extranaturelles » (E. Le Bec, 2004 : 134). C'est dans ce cadre qu'il relate le cas de Pierre De Rudder. En 1867, à la suite de la chute d'un arbre, cet ouvrier belge souffre d'une fracture ouverte de la jambe gauche. Malgré des soins " assidus et prolongés » apportés par trois médecins de Bruges, la consolidation ne s'opère pas. Une plaie gangreneuse communiquant avec le foyer de la fracture contraint le blessé à garder le lit pendant plus d'un an. En 1875, huit ans après l'accident, un examen médical révèle que " la partie inférieure de la jambe ne tient plus à la partie supérieure et est mobile en tout sens. Les deux fragments de l'os cassé sont alors distants de 0,03 m et visibles au fond d'une grande plaie en continuelle suppuration. » (G. Boissarie, 1911 : 36). Ne constatant aucune amélioration de son patient à la suite de cet examen, le Professeur Thiriart de Bruxelles considère que "toute intervention autre que l'amputation serait illusoire ". Refusant de se soumettre à cette mesure extrême, l'estropié n'a d'autres solutions que de regagner son lit. Désormais " abandonné des médecins ", il doit se résigner à endurer "d'atroces souffrances " (G. Boissarie, 1911 : 37). Le 7 avril 1875, en désespoir de cause, il part en pèlerinage au sanctuaire de Notre-Dame de Lourdes à Oostacker, en Belgique. Là-bas, alors qu'on l'a installé sur un banc pour qu'il se repose, et qu'il prie pour sa guérison, il se lève d'un bond et constate qu'il est guéri. À son retour, plusieurs médecins, qui avaient tenté pendant des années de soigner sa fracture, tiennent à l'examiner : l'os est soudé et la plaie a totalement disparu. À la tribune, le Docteur Gemelli rapporte leurs réactions : «stupéfaction ! »Celui que la médecine condamnait peut à nouveau "marcher, se tenir debout et travailler aussi bien qu'avant son accident !»(G. Boissarie, 1911 : 41). Pour terminer de convaincre 
l'assemblée, il rappelle que le 24 mai 1899, quatorze mois après sa mort, le corps de Pierre De Ruddera été exhumé afin de permettre l'extraction et l'examen médical des deux jambes.

À la fin de l'exposé du Docteur Gemelli, douze confrères lui signifient « leur attachement à la philosophie positiviste qui précisément rejette le miracle » (Le Bec, 2004 : 134). Refusant de discuter, d'autres répondent par un "silence méprisant ». D'autres encore affirment que "l'autosuggestion peut produire le cal d'une fracture, comme celle de De Rudder, par une loi physiologique à découvrir plus tard»(E. Le Bec, 2004 : 135). Beaucoup rejettent les témoignages des médecins belges en prétextant "qu'ils ont dû se tromper» (E. Le Bec, 2004 : 135). Ignorant sans doute qu'elle n'existait pas encore en 1875, un médecin réclame même une "radiographie d'avant la guérison de la jambe » (E. Le Bec, 2004 : 135). Le ton monte. Le Docteur Gemelli est accusé d'avoir « falsifié » les faits présentés. On le traite de "faussaire » (E. Le Bec, 2004 : 135). Surtout, on rit de «ce qui ne peut être qu'une comédie ", une "vaste farce », celle d'une fracture «ressoudée instantanément» (T. et G. Valot, 1956: 83). "Allons ! Avez-vous seulement examiné la bonne jambe ? " lance un médecin dans un éclat de rire général (T. et G. Valot, 1956 : 83).

Faut-il s'étonner que ces rieurs s'appliquent, par la dérision, à repousser la singularité d'une guérison qui contredit leurs connaissances médicales autant qu'elle échappe à leur vision de l'ordre des choses ? En s'attachant à dénoncer la « fraude » ou la "supercherie ", ce qu'ils rejettent ce n'est pourtant pas seulement la guérison elle-même, mais "les preuves » que leur confrère avance pour la placer au-dessus de toute incertitude et de toute objection. Ce qui est ici contesté, c'est moins la capacité à guérir du corps vivant que l'idée selon laquelle l' « autopsie du miracle " ${ }^{1}$, à laquelle certains médecins pensent s'être livrés, constituerait une "démonstration scientifique rigoureuse » susceptible de "rendre toute contestation impossible et toute objection vaine » (G. Boissarie, $1911: 18$ ).

Pourtant n'est-ce pas précisément la tâche que l'Église a confiée aux médecins du Bureau médical du sanctuaire de Lourdes dès 1883 (L. Ogorzelec, 2010) ? En écartant, dans le cadre du contrôle médical qu'ils exercent sur les guérisons déclarées "miraculeuses ", les cas qui pourraient donner lieu à des explications « naturelles » et attirer la critique des opposants aux miracles, ne livrent-ils pas aux autorités religieuses une « constatation de la matérialité du fait extraordinaire

1. À propos de ce cas, le Docteur Boissarie, président du Bureau Médical de Lourdes (de 1892 à 1917) écrira: "C'est la première fois que l'on fait l'autopsie d'un miracle, que l'on surprend pour ainsi dire le mécanisme d'une opération surnaturelle... Cette autopsie d'un miracle, c'est la chose la plus étonnante que nous possédions. Toutes les objections que l'on soulève autour des guérisons de Lourdes : les plaies nerveuses, la foi qui guérit, les effets de la suggestion, tout vient échouer devant des faits d'une évidence pareille. La démonstration a toute la rigueur d'une démonstration scientifique. » $(1911: 43)$. 
scientifiquement établi » (J.-M. Cassagnard, 1964 : 23), une matière " à garantie de résistance » suffisamment solide et stable pour affronter la polémique ${ }^{2}$ ?

Associant médecins et ecclésiastiques, ce dispositif de contrôle propre au sanctuaire de Lourdes a déjà fait l'objet d'une analyse sociologique (L. Ogorzelec, 2011). Nous voudrions ici tenter de mieux comprendre les évolutions qui ont marqué la phase médicale de cette procédure de reconnaissance en nous appuyant sur la théorie de la « conversation de gestes » de G. H. Mead (2006). Pour l'auteur de L'Esprit, le soi et la société, les gestes sont des "phases de l'acte social» (2006 : 133) qu'on ne saurait isoler les uns des autres sans perdre leur réelle signification. En d'autres termes, l'acte social est une totalité dynamique en train de se dérouler, constituée par des gestes qui se répondent les uns les autres. Et c'est en ce sens que l'acte peut être décrit comme une " conversation de gestes ": "L'attitude d'un individu, écrit Mead, provoque une réponse chez un second individu qui, à son tour, suscite de nouvelles attitudes et réponses chez le premier individu, et ainsi de suite, indéfiniment. »(G. H. Mead, 2006 : 106). Reprenant cet exemple, Mead note : Nous retrouvons une situation analogue dans le cas de la boxe ou de l'escrime. La feinte de l'un provoque la parade de l'autre, qui amène le premier à modifier son attaque, si bien qu'il peut y avoir beaucoup de mouvements dans un sens ou dans l'autre avant qu'il n'y ait une attaque. » (2006 : 132). Il faut sans doute rappeler ici que, dans ces ajustements réciproques, "certaines parties de l'acte deviennent un stimulus pour l'autre organisme qui ajuste ses réponses; lesquelles, à leur tour deviennent un stimulus pour le premier organisme, qui modifie sa propre action ou en amorce une différente » (G. H. Mead, 2006 : 132).

Dans la lignée de ce qui précède, ne faut-il pas envisager la création du Bureau médical de Lourdes comme une réponse dans une "conversation de gestes "? La procédure de reconnaissance des guérisons déclarées miraculeuses au sein de ce sanctuaire n'a-t-elle pas été construite et progressivement ajustée pour répliquer aux attaques des rationalistes, positivistes, médecins athées ou libres penseurs, "adversaires » des guérisons miraculeuses ? Ne s'est-elle pas aussi ajustée aux évolutions de la médecine et de la prise en charge thérapeutique des malades? Dans cette configuration dynamique, le concept de "conversation de gestes » n’est-il pas heuristiquement plus fécond qu'une approche plus classique, par

2. On ne peut manquer de penser ici à la manière dont des substances chimiques sont livrées par l'industrie au laboratoire avec des garanties de pureté : "Tant que les éléments chimiques ne sont pas connus avec des garanties de pureté suffisantes, garanties nettement codifiées en des critères de pureté bien coordonnés dans une synthèse de lois rationnelles, on ne peut vraiment pas, explique G. Bachelard, parler d'une chimie bien fondée. L'à-peu-près dans l'ordre de la matière interdit un rationalisme de la matière. " (1972: 8). De la même manière, concernant les guérisons déclarées au sanctuaire de Lourdes, l'Église s'interdit d'envisager la possibilité d'un "miracle» tant que les médecins du Bureau Médical et du Comité Médical International de Lourdes n'ont pas établi avec certitude leur caractère «inexplicable ». 
exemple en termes d'institutionnalisation ou de rationalisation du charisme (M. Weber, 1971) ? Appelant le chercheur à prêter attention à un dialogue du sanctuaire de Lourdes avec certaines composantes de la "société englobante ", au lieu de proposer une lecture du rôle de l'expertise médicale des guérisons comme autant d'étapes d'une rigidification visant sa seule permanence - voire sa conservation -, il permet de mettre l'accent sur de véritables répliques (au sens propre du mot) qui l'insèrent de manière dialogique dans une totalité historique plus vaste. En permettant de sauver le « contexte stimulus-réponse » dans lequel s'inscrivent les conduites et en rendant saisissable ce que l'on observe réellement (L. Quéré, 2004), la notion de " conversation de gestes " permet de préserver la complexité de l'expérience concrète des acteurs tout en donnant une clé d'intelligibilité concernant leurs façons spécifiques - toujours indexées sur la situation de s'ajuster les uns par rapport aux autres.

Dans le prolongement de ces remarques, il importe également de souligner la spécificité de notre démarche par rapport aux recherches qui ont porté sur le sanctuaire de Lourdes. À cet égard, plusieurs d'entre elles ont mis l'accent sur l'importance de la dimension politique dans le devenir d'un des plus importants lieux saints de la chrétienté. Étudiant l'histoire du pèlerinage en termes de contreculture politique hostile à la force montante de la laïcité, l'historienne R. Harris inscrit le lancement du pèlerinage national, à la fin des années 1870, dans un «vaste complot clérico-monarchique » dépassant les frontières nationales (2001: 38). De même, les travaux de E. Claverie (2003, 2008, 2009) ont permis de repenser, dans une perspective anthropologique, les phénomènes d'apparitions et les mouvements de pèlerinages qui leur sont associés, en rendant compte de la multiplicité des dimensions et des enjeux qui se nouent autour de ces "phénomènes sociaux totaux ». Il nous semble, en revanche, que la question même des « miracles de Lourdes » n'a pas bénéficié d'un tel effort de renouvellement épistémologique : comment, dans le cadre de la procédure de reconnaissance, la médecine est-elle d'abord conduite à constater une guérison ? Comment peut-elle la considérer comme « inexplicable » ? Comment, ensuite, l'Église peut-elle procéder à l'authentification et à la proclamation du miracle ? Il importe d'abord de souligner que, si depuis le $\mathrm{XI}^{\mathrm{e}}$ siècle, le magistère catholique exerce un droit de regard, allant s'élargissant, sur les miracles déclarés par les fidèles ${ }^{3}$ - soumettant ces derniers à un cadre

3. Depuis cette période, des documents pontificaux affirment la nécessité de vérifier les miracles allégués dans le cadre des procédures de canonisation par une investigation systématique. Après le XIII ${ }^{\mathrm{e}}$ siècle, cet examen devient un monopole que seul le Pape et les Évêques détiennent. Dans le prolongement de cette exigence de contrôle, et afin de centraliser la procédure d'enquête, une congrégation romaine particulière, appelée Congrégation des Rites, est constituée en 1588. Cette dernière prend exemple sur la procédure pénale de l'époque, notamment en matière de témoignage, estimant qu'il faut être au moins aussi certain pour canoniser quelqu'un que pour le condamner (P. Delooz, 1997). Dans ce contexte, les miracles sont également soumis à un cadre juridique de reconnaissance officielle de plus en plus rigoureux où l'utilisation des méthodes scientifiques complète progressivement l'enquête épiscopale (K. Woodward, 1992). On notera 
juridique de reconnaissance officielle de plus en plus rigoureux où l'utilisation des méthodes scientifiques complète progressivement l'enquête épiscopale -, c'est sans doute à la suite des événements de Lourdes ( "apparitions » et " guérisons » à partir de 1858) que cette volonté de contrôle s'exprime sous une forme nouvelle (L. Ogorzelec, 2010). On peut penser, ensuite, que les réponses aux questions précédemment formulées doivent s'appuyer sur l'analyse des évolutions des techniques de diagnostic, des progrès réalisés dans la thérapeutique et la prise en charge sociale des malades... De ce point de vue, la littérature scientifique sur les médecins et la médecine au XIX siècle (notamment: G. Canguilhem, 1975 ; P. Guillaume, 1990 ; J. Léonard, 1978) constitue une référence précieuse, permettant de construire un cadrage contextuel indispensable pour comprendre ces évolutions. En revanche, elle ne permet pas d'éclairer la manière dont ces évolutions se sont imposées aux médecins du Bureau médical de Lourdes : comment se sont-elles traduites dans l'activité des médecins du sanctuaire ? Comment ces derniers se sont-ils ajustés (au sens pragmatiste du terme) ?

À cet égard, R. Harris observe ironiquement : "Que le Bureau des Constatations médicales ait vu le jour au moment précis où la médecine parisienne atteignait le sommet de sa gloire internationale pour ses travaux sur l'hystérie n'est peutêtre pas une coïncidence !» (2001 : 438). Que cette instance médicale soit créée à un moment où les critiques rationalistes se déchaînent contre Lourdes et que son fondateur soit présenté comme un spécialiste « des troubles nerveux, des maladies imaginaires et des diverses formes de l'hystérie » (T. Mangiapan, 1994 : 83) n'est sans doute pas, non plus, un hasard. En effet, au cours de cette période, la médecine catholique doit contrer l'influence de J.-M. Charcot qui invalide toute procédure de reconnaissance miraculeuse en soutenant que les saints, martyrs et miraculés relèvent simplement de mécanismes psychopathologiques (N. Edelman, 2003). Face aux défis lancés par leurs détracteurs, ainsi qu'au regard des avancées des sciences médicales, ce sont les modalités de répliques des médecins du sanctuaire de Lourdes qu'explore cet article.

\section{De la mise en place des répertoires de guérisons à la création du Bureau médical}

Si de nombreuses guérisons sont déclarées à Lourdes depuis les visions de Bernadette Soubirous en 1858, ce n'est qu'avec l'arrivée, en 1866, des missionnaires diocésains, jusque-là chargés du sanctuaire de Notre-Dame de Garaison, que leur enregistrement devient systématique (Y. Chiron, $2000: 69$ ). En ouvrant un registre où sont notés quotidiennement les faits importants, ils font de chaque

enfin que dans le prolongement de l'évolution de cette procédure, le Vatican crée à Rome, en 1948, une Consulta Medica composée de médecins laïques chargés de déterminer si une " guérison extraordinaire ", rapportée en faveur d'un candidat à la sainteté, est "véritablement inexplicable par la science ". 
déclaration de guérison un événement enregistré, même si celle-ci ne fait pas encore l'objet d'une information contrôlée médicalement. Ce n'est qu'à partir d'avril 1868, avec la publication des Annales de Notre-Dame de Lourdes, que les chapelains imposent progressivement un cadre qui se veut plus rigoureux et qui s'appuie davantage sur la médecine. "Ne pouvant plus se satisfaire de témoignages essentiellement subjectifs, naiffs et difficilement crédibles », l'accroissement des exigences doit conduire à " une meilleure prise en charge des grâces obtenues et forcément, à un plus grand nombre de faits de guérison, plus crédibles, plus aptes à être publiés et à convaincre »(T. Mangiapan, 1994 : 71). Concrètement, cela se traduit par la volonté de recueillir des informations de plus en plus précises concernant les personnes qui se déclarent "guéries » (des renseignements sur la maladie, sur la guérison et ses conséquences...).

Parallèlement à cette recension et à la progressive construction administrative des cas de guérison, le contrôle des médecins s'organise : entre 1870 et 1880, Henri Vergez, Professeur à la Faculté de Médecine de Montpellier, procède, à plusieurs reprises, à l'examen médical de personnes qui se déclarent guéries. À l'occasion des grands pèlerinages, comme "le National »- au cours duquel les guérisons "se multiplient »-, son travail est renforcé par d'autres médecins (Y. Chiron, 2000 : 68). Mais ce n'est qu'en 1883 que le contrôle médical des guérisons devient officiel et systématique. Cette année-là, le successeur du Professeur Vergez, le Docteur Dunot de Saint-Maclou, fonde avec le Père R. Sempé, supérieur des chapelains et recteur du sanctuaire, un Bureau médical. Avec la création de cette instance permanente, on passe progressivement de l'enregistrement et de la constatation des guérisons au contrôle et à la certification. Les " cahiers " où sont retranscrits les témoignages des personnes qui se déclarent guéries sont remplacés par un "dossier » permettant une observation médicale approfondie de chaque cas de guérison.

Excluant progressivement le personnel non médical - et spécialement les prêtres, qui avaient longtemps eu la charge d'enregistrer les déclarations de guérisons -, l'orientation du Bureau médical montre clairement la progression de l'influence de la médecine dans la procédure de reconnaissance des guérisons de Lourdes. Le Docteur Dunot de Saint-Maclou, premier médecin permanent du sanctuaire, s'attache en effet à réunir autour de lui un véritable " collège médical », composé des médecins, de plus en plus nombreux, qui accompagnent les malades des pèlerinages : " De telle façon, explique-t-il, que les enquêtes à propos des guérisons puissent être, non pas le fait d'un médecin, aussi qualifié qu'il soit, mais bien plutôt un travail collectif, plus apte à permettre des conclusions objectives. » (T. Mangiapan, 1994 : 77). Cette volonté de s'ajuster à la complexité des situations qu'ils rencontrent et de mobiliser la profession médicale pour encadrer les pèlerins se traduit également par un changement de ton significatif dans la façon de considérer les guérisons. 


\section{De la guérison surnaturelle à la guérison inexplicable}

Dans la seconde moitié du XIX siècle, la tradition selon laquelle les lois de la nature sont immuables est encore vivace ${ }^{4}$. Fortement marquée par la pensée mécaniste et matérialiste $\mathrm{du} X \mathrm{XVII}{ }^{\mathrm{e}}$ siècle, la notion de nature recouvre les traits d'une totalité close. Espace d'ordre immobile et socle positif des connaissances, elle conserve la figure du cosmos. Dans cet ordre naturel, l'idée d'un Dieu capricieux intervenant dans le monde, au hasard et sans précision, est impensable. Car le caractère arbitraire d'une telle intervention ne pourrait manquer de dérégler l'ordre de la nature.

Remettant en cause la doctrine de l'Église catholique en même temps que la justification de l'autorité de Dieu, cette conception de la nature apparaît clairement comme une arme dirigée contre le rôle accordé aux initiatives ponctuelles de Dieu dans la religion chrétienne. Il faut souligner cependant qu'il s'agit d'une arme à double tranchant. Car c'est précisément cette conception que la plupart des théologiens catholiques et, d'une manière générale, les esprits religieux vont utiliser contre la menace d'un rationalisme résolument matérialiste. De leur point de vue, si le "naturel " est ce qui arrive conformément aux lois de la nature, le "surnaturel » sera ce qui se situe en dehors, ce dont on ne peut rendre raison en termes de lois. À cet égard, l'ordonnance de Monseigneur Laurence qui ouvrait l'enquête officielle sur les faits de la Grotte de Lourdes en juillet 1858 posait le problème en ces termes : " ces faits sont-ils surnaturels ? Les guérisons opérées peuvent-elles s'expliquer naturellement ou peuvent-elles être attribuées à une cause surnaturelle ${ }^{5}$ ? ». La rupture avec l'autosuffisance de la nature que les hommes de science affirment alors, susceptible d'épuiser par un nombre restreint de lois toutes les possibilités d'une approche rationnelle des phénomènes, rend plus éclatant l'écart à ces lois et tout ce qui leur échappe.

Dans une telle perspective, la "soudaine initiative créatrice » ${ }^{6}$ d'une divinité qui dévoie le cours ordinaire des choses, accule le médecin à reconnaître les possibilités restreintes de la nature. La «facilité », la " promptitude », l' " instantanéité » (B. Billet, 1976), en d'autres termes, la violation et le bouleversement des prévisions thérapeutiques et la contradiction des préceptes et des prévisions de la science médicale constituent, pour le Professeur Vergez, les critères essentiels du surnaturel. En tant qu'expert chargé de contrôler les premières guérisons de

4. «C'est une simple induction, une expérience millénaire, qui a imposé au chercheur la certitude toujours croissante et finalement définitive de l'immuabilité des lois de la nature, au point que cette grande vérité ne saurait soulever le moindre doute. " (L. Freuler, 1997: 65 ; R. Lenoble, 1969).

5. Ordonnance de Monseigneur Laurence, Évêque de Tarbes, 28 juillet 1858, intégralement retranscrite in R. Laurentin, $1958: 210-214$.

6. «Rapport du Professeur Vergez sur les guérisons attribuées à l'eau de la Grotte de Lourdes et enregistrées par la commission épiscopale ", avril 1860, intégralement retranscrit dans R. Laurentin, 1959. 
Lourdes, il délivre, par exemple, les notations suivantes : " cette guérison se classe dans l'ordre surnaturel ", " ces faits s'inscrivent dans un ordre de faits supérieurs aux puissances de la nature » (Billet, 1976), etc. Ramenant certains faits observés à ce-qui-surpasse-la-nature, dans cette expertise médicale le passage du naturel au surnaturel, du connu à l'inconnaissable, ne contredit nullement les tendances empiristes du médecin : la transparence de la nature suppose au contraire le renvoi de l'inexplicable dans la surnature selon le compromis alors en place : "S'il y a un fait qui diffère de tous les autres et nous place au seuil d'un autre monde que le monde accessible à la Science proprement dite, c'est être fidèle à la Science que de savoir, en reconnaissant ce fait, dépasser la Science. » (J.-M. Cassagnard, 1964 : 22). Dans cette perspective, le Professeur Vergez attribue certaines guérisons de Lourdes à une cause surnaturelle en traçant lui-même la limite de la région où s'étend son savoir. Il admet le surnaturel comme une région de faits qui jouxte son propre domaine, reconnaissant dans le théologien le propriétaire de fiefs qui délimitent les siens (M. de Certeau, 2005 : 225). La profession de foi qu'il signe en 1886 va pleinement dans ce sens : "On me demande ce que j'ai vu à Lourdes, deux mots suffisent pour le dire : par l'examen des faits les plus authentiques, placés au-dessus du pouvoir de la science et de l'art, j'ai vu, j'ai touché l'œuvre divine, le miracle. " (cité in Annales NDL, 1888-1889 : 259). Mais, au moment où le Professeur Vergez écrit ces lignes, son successeur le Docteur Dunot de Saint-Maclou, en place au Bureau médical de Lourdes depuis déjà deux ans ${ }^{7}$, formule à l'égard des cas de guérisons des conclusions médicales bien différentes. Le changement de vocabulaire est significatif : on ne trouve plus de "guérisons surnaturelles ", mais des "guérisons inexplicables en l'état des connaissances actuelles ». À l'inverse de son prédécesseur, loin de poser une limite à la raison pour constituer le domaine où s'exerce la connaissance, il englobe à l'avance dans une causalité naturelle tout le connaissable. L'inconnu et l'étrange ne sont plus renvoyés à un "en dehors de la nature », mais prennent place dans l'avenir de la connaissance " naturelle », représentant ce que n'a pas encore atteint le pouvoir de la raison. Du surnaturel à l'inexplicable : comment expliquer un tel renversement dans l'interprétation des cas de guérisons? Une connaissance historique, même sommaire, de l'état de la recherche et de l'enseignement en médecine, en France, à la fin du XIX ${ }^{\mathrm{e}}$ siècle, autorise à penser que ce changement n'est pas imputable à une simple divergence de sensibilités singulières concernant l'interprétation des guérisons de Lourdes. Il est le symptôme d'un changement épistémologique de plus grande ampleur marquant le passage de la médecine hippocratique à la médecine expérimentale (G. Canguilhem, 1975).

7. À partir de 1884, c'est lui qui, en tant que «docteur annaliste de la Grotte », présente les cas de guérisons dans les Annales de Notre-Dame de Lourdes, publiant à la suite des relations et des certificats des notices où il discutait les cas présentés. 


\section{Le passage de la médecine hippocratique à la médecine expérimentale}

Jusqu'à la seconde moitié du XIX ${ }^{e}$ siècle, la connaissance médicale se fonde sur l'observation. Elle se caractérise par une passivité devant un réel considéré comme autonome et auquel la connaissance doit s'adapter. Les systèmes nosologiques découpent dans l'expérience un champ de savoir possible, définissent le mode d'être des objets qui y apparaissent et arment le regard de pouvoirs théoriques. Dans cette perspective, «le médecin cherche à remplir ce qu'il sait avec ce qu'il voit. Il quête la manifestation de ses concepts nosologiques et verse dans la simple reconnaissance qui n'accepte du visible que le connu. » (M. de Certeau, 2005 : 221). La série indéfinie des faits que le regard balaie s'articule avec la série finie des catégories médicales que détient alors la connaissance médicale. Ainsi, même si, comme le souligne G. Canguilhem, Cabanis « utilise constamment les termes de fait, d'observation, d'examen, d'expériences, de comparaison, d'empirisme rationnel, jamais celui d'expérimentation ne vient sous sa plume. [Et] la Réforme, dont il expose le projet, à l'aube du XIX siècle, ne tend en rien à faire de la médecine autre chose qu'une science d'observation. » (G. Canguilhem, 1975 : 129).

C’est précisément cette position que la "médecine expérimentale », notamment portée par C. Bernard, cherche à dépasser dès $1854^{\circ}$. Contre une médecine passive, contemplative et descriptive, préoccupée avant tout de définitions et de classifications, il promeut une "science conquérante " où "l'homme devient un inventeur de phénomènes, un véritable contremaître de la création ; et [où] l'on ne saurait, sous ce rapport, assigner des limites à la puissance qu'il peut acquérir sur la nature par les progrès futurs des sciences expérimentales. » (C. Bernard, 1945 : 71). Dans la perspective d'une science " progressive » et " prométhéenne », lancée à l'assaut de l'inconnu, cherchant avant tout dans le réel ce qui contredit ses connaissances, la notion de "surnature » s'évanouit. En effet, loin de poser des limites, "un expérimentateur est toujours au niveau du progrès; il sacrifie autant de théories qu'il faut pour avancer. » (C. Bernard, 1947 : 179). Dans une telle perspective, la guérison inexplicable n'est plus cette chose qui se trouve à jamais " hors d'atteinte de la raison scientifique " pour prendre place dans le domaine $\mathrm{du}$ «surnaturel ». Elle devient au contraire une cible pour la raison; l'altérité qui doit alerter la pensée ; l'objet sur lequel il faut concentrer la lumière. Dès lors, l'inexplicable n'est plus qu'un futur explicable inscrit dans la promesse d'un progrès scientifique indéfini.

8. «Claude Bernard enseigne publiquement pour la première fois la spécificité de l'expérimentation en physiologie, dans la Leçon du 30 décembre 1854, la troisième du cours qu'il donne pour la dernière fois, comme suppléant de Magendie au Collège de France, sur la physiologie expérimentale appliquée à la médecine. »(G. Canguilhem, 1975 : 145). 
En s'engageant dans une telle voie, C. Bernard exprime non seulement une interprétation possible de certains résultats de la médecine de son temps, mais aussi un ensemble théorique bien plus vaste. En effet, à l'époque où il fonde les principes de la médecine expérimentale, les physiciens élaborent le concept d'entropie ; C. Darwin et A. R. Wallace découvrent que la vie, loin d'être fixée une fois pour toutes, relève de l'évolution. Or, pour constituer le garant « scientifique » que sollicite l’Église, le médecin qui intervient dans le cadre du contrôle des guérisons de Lourdes n'est-il pas, plus que tout autre, tenu de s'inscrire dans la lignée de ces avancées scientifiques, de se conformer aux évolutions des connaissances médicales ainsi qu'à l' " ordre du discours" de sa discipline ? Les autorités religieuses ne lui demandent-elles pas précisément de parler " en médecin ", comme un capitaine demande à ses éclaireurs, avant la bataille, de le renseigner sur les positions de l'ennemi ? Soucieuses de crédibiliser les guérisons de Lourdes, les autorités religieuses ne peuvent se satisfaire d'une forme d'expertise reposant sur une tradition contestée dans le champ médical. Et on comprend, dans cet ordre d'idée, que si le Professeur Vergez pouvait encore se fonder sur des certitudes inaccessibles au doute en 1860, le Docteur Dunot de Saint-Maclou, en 1883, à dû tourner la page qui prétendait lier la reconnaissance d'une guérison miraculeuse à une certitude médicale définitive et dû poser, en des termes différents, ses constatations conclusives. Conformément aux évolutions des connaissances, il ne peut donc plus être question, au niveau médical, de guérisons "contraires aux lois naturelles ». Elles deviennent seulement " inhabituelles ", " imprévisibles ", " inexplicables », et même « inexplicables dans l'état de la connaissance médicale ».

\section{La stratégie offensive des médecins catholiques}

Face à l'évolution de la médecine, l'ajustement proposé par le Docteur Dunot de Saint-Maclou n'était qu'une première réplique caractérisant une position réactive que les médecins du Bureau médical allaient dépasser. À la mort de ce dernier, Gustave Boissarie, un médecin de Sarlat, prend la direction du Bureau médical et y demeure attaché de 1892 à 1917. Durant cette période, le discours sur les guérisons change notablement. Les termes que le Docteur de Saint-Maclou prenait soin de ne jamais prononcer dans le cadre de ses fonctions - les jugeant inadéquats et irrecevables - reviennent régulièrement sous la plume du Docteur Boissarie. Ainsi, après les contrôles médicaux, certaines guérisons sont à nouveau qualifiées de «miraculeuses » et de «surnaturelles »- la reconnaissance par les médecins du Bureau médical étant alors considérée, à elle seule, comme suffisante pour arriver à pareille conclusion.

La compréhension de ce revirement (de ce « geste ») - que certains responsables ultérieurs du Bureau médical n'hésiteront pas à qualifier de "véritable régression » (T. Mangiapan, 1994) - n'est possible que si on l'envisage comme une réplique dans une « conversation de gestes » où s'opposent, pendant cette période, catholiques et libres penseurs. Dans un article traitant de la transformation des foules en 
processions à Lourdes en 1858, nous avons montré quelles controverses, dressant croyants contre rationalistes, a suscité "l'affaire de la Grotte » (L. Ogorzelec, 2010). Les rumeurs d'apparitions, puis de guérisons, rapidement relayées par la presse, ont secoué tout le pays jusqu'aux instances politiques les plus élevées. Avec le triomphe définitif des républicains dans les années 1879-1880, la lutte, loin de s'affaiblir, semble s'intensifier. Les scientifiques volontiers "scientistes" se présentent comme les " prêtres de la vraie religion ${ }^{9}$ ", et affirment avoir purgé le monde de ses mystères ${ }^{10}$ au moment même où d'autres proclament la faillite et la «banqueroute de la science ${ }^{11}$ ».

À la fin $\mathrm{du} \mathrm{XIX}^{\mathrm{e}}$ siècle, les sociétés de Libre Pensée reprennent la parole, commencent à se structurer et à s'organiser. Plusieurs fédérations existent conjointement ${ }^{12}$ : la Société pour la Propagation de la Foi civile (1878), l'Union Démocratique de Propagande anticléricale (1880), la Ligue anticléricale (1881), la Fédération Française de la Libre Pensée (1890) etc. Résolument engagées du côté de la science et du progrès, ces sociétés tiennent un discours militant et se sont assigné un but: "convaincre le plus grand nombre de renoncer aux superstitions, aux préjugés de la foi et de rallier la cause lumineuse et émancipatrice de la raison et de la science " (J. Lalouette, 1989 : 21).

Face à l'émergence de ces fédérations, des efforts sont déployés par le catholicisme. Au moment même où le Bureau médical est fondé à Lourdes, on observe en effet une renaissance religieuse très marquée dans le milieu médical. D'une part, le Docteur Le Bèle, chirurgien du Mans et grande figure de la médecine chrétienne de la fin du XIX ${ }^{\text {e }}$ siècle, organise, en septembre 1884, la Société médicale de Saint-Luc, Saint-Côme et Saint-Damien ${ }^{13}$. D'autre part, la Faculté catholique de Lille voit, au même moment, ses premiers docteurs prendre place dans le corps médical français (P. Guillaume, 1990 : 93). Naissant à la même heure, ces trois œuvres catholiques vont se développer ensemble et, à l'époque de la construction de la République laïque, se prêter un mutuel appui (H. Guillemain, 2003).

9. Dans L’avenir de la science, rédigé en 1848, et publié en 1890, E. Renan se présente comme "le prêtre de la vraie religion".

10. M. Berthelot, célébrant le pouvoir de la science, n'avait pas hésité à affirmer en 1885 , dans la Préface de son ouvrage sur Les origines de l'alchimie, que "le monde est aujourd'hui sans mystère ".

11. F. Brunetière conféra à cette formule une notoriété toute particulière grâce à un article retentissant, intitulé "Après une visite au Vatican ", qui parut dans La Revue des Deux Mondes en janvier 1895 .

12. Avant leur unification en 1912 donnant naissance à une Union Fédérative des Libres Penseurs de France.

13. Concernant l'évolution des effectifs et les éléments géographiques de la Société médicale Saint-Luc, H. Guillemain souligne « la relative marginalité des médecins du Sud, du Centre et de l'Est. En 1899, un tiers des médecins adhérents font partie d'un comité de l'Ouest, près d'un médecin sur cinq est parisien, un sur cinq lillois, un sur six lyonnais. La géographie de la médecine catholique ne se modifie guère après 1900 » (H. Guillemain, 2003). 
D'ailleurs, est-il nécessaire de rappeler ici que les quatre premiers présidents du Bureau médical de Lourdes, nommés par l'Évêque de Tarbes, seront choisis parmi les adhérents de la Société Saint-Luc, Saint-Côme et Saint-Damien (le Docteur de Saint-Maclou, président de 1883 à 1891 ; le Docteur Boissarie de 1892 à 1917 ; le Docteur Cox [intérimaire] de 1917 à 1920 et le Docteur Le Bec, de 1920 à 1923) ?

À la même période, la stratégie offensive développée par les médecins catholiques commence à être théorisée par une partie des apologistes catholiques afin de répondre aux objections de la science positive. Le miracle, objet déjà controversé au sein de l'Église en cette fin de XIX siècle par les aspirations réformatrices des clercs et des laïcs visant la libre recherche intellectuelle et proposant une apologétique appuyée sur les méthodes historico-critique (E. Poulat: 1996 ; F. Chaubet : 2008), devient ainsi l'enjeu d'un débat à l'extérieur de l'Église catholique, dans le conflit externe entre christianisme et modernité. À cet égard, l'abbé Mano écrit en 1899 : "Nous cherchons à démontrer que le miracle est possible et constatable, et qu'une fois constaté, il est un signe certain de la révélation. Mais nous croyons indispensable de démontrer cette possibilité du miracle, par des arguments que nos adversaires puissent entendre. " (cité in F. Rodé, 1965 : 10-11). Au même moment, les écrits de Monseigneur de Saint-Projet vont dans le même sens : "Aujourd'hui, écrit-il, les théories purement rationnelles n'occupent point le front de bataille ; ce sont les sciences expérimentales, les sciences physiques et naturelles qui troublent les consciences et semblent mettre la foi en péril. C'est avec ces mêmes armes qu'il faut combattre les ennemis de la foi. Voilà les richesses nouvelles, les forces vives, le formidable arsenal dont il faut s'emparer. " (cité in F. Rodé, 1965 : 27).

C'est dans ce contexte idéologique et politique conflictuel, où se prépare la loi concernant la séparation des Églises et de l'État ${ }^{14}$ et où l'anticléricalisme constitue le ciment dur de l'idéologie républicaine, que le Docteur Boissarie prend, en 1892, la direction du Bureau médical. Mais si, comme nous l'avons vu, la réponse des catholiques aux attaques qu'ils subissent tente d'associer rénovation théologique et appel à la science médicale, nous allons voir que la bataille apologétique se réalisera autour des phénomènes de suggestion et d'hystérie, clé de voûte de la critique des adversaires des miracles.

14. Le 9 décembre 1905, le député socialiste Aristide Briand fait voter la loi concernant la séparation des Églises et de l'État, mettant fin au Concordat napoléonien de 1801 qui régissait les rapports entre le gouvernement français et l'Église catholique. 


\section{Les guérisons de Lourdes et la question de l'hystérie}

En 1892, l'année même de sa prise de fonction, le Docteur Boissarie reçoit la visite d'un pèlerin d'un genre nouveau : "J'hésitai longtemps, écrira-t-il, à recevoir Zola dans le bureau des médecins. Le secret médical nous lie vis-à-vis de nos malades. Pouvais-je, devant un romancier et devant sa cour de journalistes, interroger des femmes et des jeunes filles, mettre à nu leurs infirmités ? Je n'en avais pas le droit. Mais devant la pression de l'opinion, il me fallut pourtant céder [...] On disait que cet homme avait trouvé son chemin de Damas, qu'il allait se convertir. » (G. Boissarie, 1911 : 26). Or, si Zola s'est converti, ce n'est pas au catholicisme ! La thèse que l'écrivain défend est déjà clairement exposée dans les pages du Docteur Pascal dont il achève l'écriture l'année de sa visite à Lourdes : " L'avenir de l'humanité est dans le progrès de la raison par la science. »(E. Zola, 2004 : 102).

Dans le grand combat qui divise, en cette fin de siècle, la plupart des intellectuels, et que les événements de Lourdes symbolisent, Zola a donc déjà choisi son camp. De ce fait, il n'était pas difficile de présager que, débordant le champ romanesque, l'écriture de Lourdes se trouverait d'emblée subordonnée à un plus vaste projet moral : " mettre en scène, l'espace d'un pèlerinage, le grand drame idéologique dont Lourdes représente un des points critiques : la lutte de l'esprit de foi contre l'esprit de raison "(J. Noiray, 1995 : 11). Ainsi, c'est à la lumière d'une psychologie fondée sur les enseignements de J.-M. Charcot et sur une sociologie qui retrouve les postulats de G. Tarde sur le comportement mimétique des foules, que Zola dépeint l' « irraison collective » des masses "suggestionnées » dans le piétinement des processions. Dans ce tableau, les guérisons miraculeuses ne sont plus que des maladies nerveuses mal étudiées ; l' "inconnaissable " n'est que l' " inconnu » que la science, patiemment, s'obstine à réduire. Le roman paraît en 1894. Incendié dès sa sortie par la presse conservatrice et religieuse, il est mis à l'Index, mais son grand succès en librairie ne manque pas de déclencher chez les catholiques un sentiment de persécution et de conspiration.

Ce sentiment est renforcé par le fait que l'année même où Zola séjourne à Lourdes, J.-M. Charcot publie, dans La Revue hebdomadaire de décembre, son article "La foi qui guérit " dans lequel il défend l'hypothèse que les guérisons supposées "miraculeuses " ne s'appliquent qu'aux manifestations physiques de l'hystérie ${ }^{15}$. Là encore, le Bureau médical de Lourdes et son protocole de contrôle des guérisons sont explicitement visés. Mais la réplique ne se laisse guère attendre.

15. «Je sais bien qu'aujourd'hui, écrit-il, des médecins préposés à la constatation des miracles s'appliquent à montrer que des tumeurs, des ulcères parmi les plus rebelles, sont monnaie courante dans le domaine de la thérapeutique miraculeuse. Je ne le nie pas : je pense comme eux que la faith-healing (la foi qui guérit) peut directement faire disparaître, dans certains cas, des ulcères et des tumeurs ; [...] mais s'il était démontré que ces tumeurs et ces ulcères autour desquels on mène tant de bruit sont de nature hystérique, justiciables de la même faith-healing que les convulsions et les paralysies, c'en serait donc fini du miracle. Pourquoi alors jeter tant de défis à la face de la science, qui finit, en somme, par avoir le dernier mot en toutes choses ? " (J.-M. Charcot, 1892 : 112-132). 
Elle consistera à faire du Bureau médical de Lourdes un « espace scientifique de rigueur médicale » offrant l'opportunité d'un vaste champ d'observation clinique (N. Edelman, $2003: 223$ ).

\section{Créer une " société savante "}

Le Docteur Boissarie connaît bien le monde hospitalier parisien (N. Edelman, 2003). Il a travaillé à l'hôpital de la Charité où il a fait une partie de son internat. "J'ai eu l'avantage, écrit-il, d'être l'interne de Briquet et de recueillir les derniers échos de son enseignement, j'ai appris à son école que l'hystérie peut reproduire tous les troubles fonctionnels, emprunter les allures et les symptômes de toutes les maladies, sans arriver jusqu'à la destruction de l'organe. » (G. Boissarie, 18951896 : 108). Attentif aux travaux de la Salpêtrière où il a suivi les expériences de Luys, il dit être, comme son prédécesseur, prémuni contre l'imitation et la suggestion (N. Edelman, 2003 : 216). Loin de nier les guérisons hystériques, il les écarte le plus simplement du monde en les présentant comme "naturelles » et, de ce fait, ne relevant pas du recensement du Bureau médical.

En effet, pour neutraliser et rejeter les attaques des adversaires des miracles, les médecins du Bureau médical sont explicitement chargés par l'autorité ecclésiastique de faire la distinction entre les guérisons " hystériques » et celles relevant de véritables troubles "organiques ", de trier les véritables guérisons inexplicables susceptibles de convaincre les sceptiques en rejetant les fausses déclarations de guérisons qui risqueraient de jeter le discrédit sur le lieu saint. Cette consigne n'est pas une réalité isolée qui ne concernerait que le fait des guérisons de Lourdes, mais révèle une position profondément ancrée de l'Église à cette époque. En effet, à mesure que la médecine affine son approche clinique des phénomènes de possession, de convulsions, d'extases et de visions, les faisant ainsi passer du territoire de la théologie à celui de la thérapeutique, l'Église cherche à s'en décharger en les disqualifiant fortement dans la piété chrétienne. M. Foucault a très bien décrit certaines répliques de cette " conversation de gestes " dans l'un de ses cours au Collège de France : «Lourdes répond à Loudun. Vers les années 1870-1890, il y a d'un côté Lourdes, qui dit : Les diableries de Loudun étaient peut-être, en effet, des hystéries à la manière de la Salpêtrière. Laissons à la Salpêtrière les diableries de Loudun. Mais ceci ne nous touche en rien, puisque nous ne nous occupons plus que des apparitions. Ce à quoi la Salpêtrière répond : Ce que Loudun et Lourdes ont fait, nous pouvons aussi bien le faire. Nous faisons des convulsions, nous pouvons faire aussi des apparitions. Ce à quoi Lourdes rétorque : Guérissez tant que vous voudrez. Il y a un certain nombre de guérisons que vous ne pourrez pas faire et que nous ferons. "(M. Foucault, 1999 : 210). Les interlocuteurs et certains arguments de ce dialogue reconstruit à grands traits sont sans aucun doute bien identifiés par M. Foucault. Il convient cependant de se livrer à une analyse un peu plus précise du dialogue réel. 
C'est bien dans cette perspective que, d'une manière générale, les différents responsables du Bureau médical mettront systématiquement l'accent sur « des retours à la santé en l'absence de toute médication appropriée », sur le caractère "imprévisible» des guérisons constatées et, surtout, sur la "suppression de la convalescence » qui constitue, selon eux, « la preuve palpable de l'extraordinaire dans la guérison» (J.-M. Cassagnard, 1964 : 50). Le Docteur Boissarie explique ainsi : "On voit guérir instantanément à Lourdes, des tubercules pulmonaires, des caries tuberculeuses de la colonne vertébrale, des péritonites tuberculeuses, disparaître des ulcères, des tumeurs cancéreuses... Or, ce sont des lésions matérielles des tissus organiques, sur lesquelles la suggestion est sans effet. Qui donc, conclut-il, oserait comparer ces pauvres malades qui touchent aux dernières limites de la résistance à ces fascinées de la Charité qui, le sourire sur les lèvres, s'endorment en fixant un miroir à alouettes ? (G. Boissarie, 1911 : 94). Pour s'ajuster, les médecins de Lourdes effectuent un retour aux critères canoniques, font le tri, tentent d'évacuer les hystériques, s'attachent à ne conserver que les guérisons organiques. Cette "purification " s'intègre à une réponse globale assez offensive.

Ainsi, les critiques avancées par les opposants aux miracles sont intégrées dans le fonctionnement du Bureau médical et même utilisées afin de renforcer la crédibilité des guérisons, de les rendre "plus éclatantes » encore : "Les représentants de la science officielle, explique le Docteur Boissarie, ont posé des principes qui devaient se retourner contre nous, mais que nous avons repris à notre profit en les acceptant comme base de nos jugements. » (1911: 96), mais il va encore plus loin. Si l'hystérie constitue l'occasion d'une attaque c'est aussi celle d'une opportunité pour les médecins du sanctuaire: "Les maladies nerveuses! écrit-il, nulle part on ne les connaît, on ne les étudie mieux qu'à Lourdes ! » (1911 : 83). «À côté des formes légères, à peine accusées, poursuit-il, vous avez les accidents les plus graves. C'est une clinique sans rivale et par le nombre et par la variété des sujets. » (1900: 451).

Dans la «conversation de gestes » qui se noue entre les médecins du sanctuaire et leurs contradicteurs, la question de l'hystérie devient un enjeu extrêmement important. Car si, à Lourdes, on se gardait bien jusqu'à présent de mettre en avant ce phénomène, c'est précisément sur ce terrain que le Docteur Boissarie tente de rediriger les forces du Bureau médical en estimant pouvoir former sur cette question une troisième école - l'école de Lourdes - à côté de celles de la Salpêtrière et de celle de $\mathrm{Nancy}^{16}$ (N. Edelman, 2003 : 218). Il veut créer une

16. L'École de Nancy est, avec celle de la Salpêtrière, l'une des deux grandes écoles françaises ayant contribué à l'étude des phénomènes de suggestion et d'hypnotisme. À la fin des années 1880, la querelle qui oppose l'École de Nancy à l'École de la Salpêtrière est au cœur de tous les débats sur la nature de l'hypnose, les partisans du Professeur Hippolyte Bernheim voyant dans l'hypnose un simple "sommeil » produit par la suggestion et susceptible d'applications thérapeutiques et ceux de Jean-Martin Charcot considérant que l'hypnose est un état pathologique spécifique propre aux hystériques. 
« société savante " susceptible de résister aux assauts d'un environnement hostile, capable de produire des affirmations inattaquables et de fournir " une méthode irréprochable ", de façon à ce que " les grands miracles de Lourdes ne soient plus niés que par quelques hommes d'une mauvaise foi évidente » (G. Boissarie, 1911 : 8). Aux détracteurs, il n’hésite donc pas à rétorquer : "Au lieu de se moquer du spectacle de Lourdes, les médecins devraient profiter de ce champ incomparable d'observation. Venez examiner nos malades! Jamais clinique n'a été ouverte comme la nôtre à tous les médecins qui se succèdent pendant toute la durée des Pèlerinages et dont le nombre s'accroît chaque année. Nous n'avons jamais cessé, rappelle-t-il, de prendre contact avec la science contemporaine. » (G. Boissarie, $1911: 10)$.

À défaut de pouvoir rendre la contestation impossible, le médecin de Lourdes cherche à la rendre extrêmement coûteuse. Pour y parvenir, il ne faut pas rompre le dialogue, mais au contraire forcer les adversaires à s'ajuster et à positionner leurs critiques sur un nombre restreint d'objets nécessitant la maîtrise de la médecine et la connaissance des répliques précédentes. Imposer la " conviction », rendre le miracle " indiscutable ", " incontestable ", " inattaquable »... Ces mots reviennent, obsédants, dans ses écrits, puis dans ceux de ses successeurs (notamment chez les Docteurs Le Bec et Vallet), dans les rapports et les comptes rendus publiés dans les Annales de Notre-Dame de Lourdes, enfin, à partir de 1928, dans les Bulletins de l'Association Médicale de Notre-Dame de Lourdes.

Si l'histoire de la procédure de reconnaissance des miracles de Lourdes est celle d'une constante réforme, c'est parce qu'elle s'inscrit dans une " conversation de gestes ", c'est-à-dire un ensemble de répliques entre des protagonistes en lutte. En suivre les étapes, c'est prendre la mesure de la réactivité des médecins du sanctuaire lorsqu'ils répondent aux évolutions de la médecine, aux arguments de leurs adversaires. En effet, les modifications apportées dans la construction des critères de contrôle ou dans la formulation des conclusions médicales semblent constituer autant d'ajustements aux variations de leur environnement. Dans le contexte extrêmement polémique de l'expertise médicale des guérisons déclarées « miraculeuses ", les "gestes » des médecins s'inscrivent le plus souvent dans la perspective d'une défense, d'une offensive ou d'une contre-offensive cherchant à modifier ou à bouleverser une situation qui peut leur être défavorable. À cet égard, les répliques des uns et des autres ne peuvent s'analyser que comme " enchâssées dans des ensembles de conditions eux-mêmes antérieurs affectant la situation présente et qui sont affectés par ces actions - les conséquences présentes de l'action devenant à leur tour des conditions pour les actions à venir » (A. Strauss, 1992 : 21). De ce point de vue, on ne peut comprendre, par exemple, les positions radicales qu'à pu prendre le Docteur Boissarie lorsqu'il était à la tête 
du Bureau médical, si l'on ne prend pas en considération la visite de Zola à Lourdes et la publication de l'article «La foi qui guérit » de J.-M. Charcot. N'est-ce pas dans la perspective d'une "réplique » ${ }^{17}$ que les médecins de Lourdes intégreront les critiques de leurs détracteurs dans le fonctionnement du Bureau médical et qu'ils les utiliseront afin de renforcer la crédibilité des guérisons?

Faisant un pas de plus, en nous appuyant sur G. H. Mead, nous pourrions dire que le Bureau médical apparaît comme une organisation complexe de réponses ${ }^{18}$ fragiles et faillibles, s'élaborant dans un cours d'action donné ou en fonction d'une action passée. Dans cette perspective, l'introduction des médecins spécialistes dans la procédure de contrôle médical grâce à la création du Comité médical national de Lourdes en 1947 ne peut-elle pas être comprise comme une " réponse anticipée » à une possible nouvelle attaque des adversaires du miracle ? En effet, face à une évolution perceptible dans la répartition de la compétence médicale et avec son recrutement presque exclusif en médecins généralistes, le Bureau médical dispose-t-il encore d'une compétence suffisante ? Ses conclusions peuvent-elles être tenues pour valables dans un domaine où les connaissances semblent s'affiner en même temps qu'elles se redistribuent? Les médecins de Lourdes adoptent une position de garde ${ }^{19}$, anticipant toute critique sur la fiabilité et la rigueur du contrôle des guérisons. Ne pourrions-nous pas, à propos de leur réponse, citer la remarque de G. H. Mead : " en escrime, parer c'est interpréter la botte » (2006: 161) ? Dans la " conversation de gestes » qu'ils entretiennent avec leurs détracteurs, en suivant les réajustements permanents qu'ils mettent en œuvre, les médecins de Lourdes s'efforcent de se maintenir «dans le vrai » du discours médical de leur époque (M. Foucault, 1971 : 37). Et pour ce faire, ne pratiquent-ils pas une sorte de tactique, apparentée à l'aïkido, qui détourne la force du puissant au bénéfice du faible?

À cet égard, l'ajustement le plus remarquable consiste sans doute dans le repositionnement des médecins du Bureau médical concernant ce qui fut au fondement de leur activité de contrôle : la distinction entre "maladies organiques" et "maladies fonctionnelles ". En effet, dès la fin des années 1950, la nécessité de maintenir un tel clivage commence à être rediscutée entre les médecins du sanctuaire et des différentes instances de la procédure de reconnaissance. Représentant ces derniers lors du Congrès Mariologique international de 1958 à Rome, le Professeur Salmon s'interroge : «L'intrication de l'organique et du fonctionnel est admise par tous. Celui qui la nierait passerait pour un sot ou un ignorant.

17. Le terme « réplique » est à la fois théâtral et militaire, et il n’est pas utilisé sans supposer l'existence de "frictions" (pour parler comme les militaires). On peut envisager, en termes de stratégie, sans exclure aucun rapport de forces, l'enchaînement des répliques dans la conversation par gestes.

18. C'est pour G. H. Mead la définition même de toute institution (2006:311).

19. En escrime, la garde est la position la plus favorable que prend le tireur afin d'être prêt à la défensive, à l'offensive ou à la contre-offensive. 
Puisque nous savons, poursuit-il, que toute fonction est dépendante de l'organique, pourquoi refuser les guérisons fonctionnelles avec lésions apparentes ou inapparentes ? Pourquoi refuser à Dieu le droit d'agir sur le "mental”, sur le psychisme et même sur l'esprit de l'Homme, alors qu'IL est l'Esprit Infini ? Il me semble qu'il conviendrait de réviser notre optique. »(M. Salmon, 1959). Dans la lignée de ce qui précède, on peut souligner qu'actuellement, les médecins du sanctuaire n'hésitent pas à parler de "guérison intérieure ", de " délivrance " et de "libération " en relation avec "la paix du cœur retrouvée » (colloque, Lourdes, 2004). "Nous vivons, explique l'un d'entre eux, dans des sociétés où le stress est élevé et où les conditions de travail sont parfois déshumanisantes, que l'on soit cadre supérieur ou travailleur manuel. Sans parler de la crise d'identité du demandeur d'emploi de longue durée. Bref, il existe une recherche d'équilibre personnel qui va bien au-delà de la santé au sens strict, si bien qu'il y a de nombreuses personnes qui ne souffrent d'aucune pathologie reconnue par la médecine ou la Sécurité sociale, mais qui ont une demande d'aide médicale, de soutien thérapeutique. " (colloque, Lourdes, 2004 : 91). La guerre contre les théoriciens de l'hystérie est terminée, mais le Bureau médical de Lourdes n'a sans doute pas fini de dialoguer avec son environnement.

Laetitia OGORZELEC Université de Franche-Comté LASA-UFC (EA 3189) laetitia.ogorzelec@gmail.com

\section{Bibliographie}

AnNales de Notre-Dame de LOURDes, 1888-1889.

BACHELARD Gaston, 1972, Le matérialisme rationnel, Paris, Presses universitaires de France. BERNARD Claude, 1945, Introduction à l'étude de la médecine expérimentale, Genève, Éditions du Cheval ailé.

-, 1947, Principes de médecine expérimentale, Paris, Presses universitaires de France. Berthelot Marcellin, 1885, Les origines de l'alchimie, Paris, Georges Steinheil Éditeur. Billet Bernard, 1976, "Notes d'histoire : la fonction des médecins vis-à-vis des faits de Lourdes (1858-1893) », Bulletin de l'AMIL, nº 173.

BoIsSARIE Gustave, 1895-1896, " le surnaturel et la science ", Annales de Notre-Dame de

Lourdes.

-, 1900, Les grandes guérisons de Lourdes, Paris, C. Douniol.

-, 1911, Lourdes, les guérisons, Paris, Maison de la Bonne Presse.

Canguilhem Georges, 1975, Études d'histoire et de philosophie des sciences, Paris, J. Vrin.

CASSAgnard Jean-Marie, 1964, Carrel et Zola devant le miracle à Lourdes, Lourdes, œuvre de la Grotte.

Charcot Jean-Martin, 1892, «La foi qui guérit », Revue hebdomadaire, Paris, 7, p. 112132.

Chaubet François, 2008, Catholicisme et Monde moderne aux XIX et XX ${ }^{e}$ siècles. Autour $d u$ «Modernisme ", Dijon, Éditions Universitaires de Dijon. 
Chiron Yves, 2000, Enquête sur les miracles de Lourdes, Paris, Perrin.

Claverie Élisabeth, 2003, Les guerres de la Vierge. Une anthropologie des apparitions, Paris, Gallimard.

-, 2008, Le monde de Lourdes, Paris, Gallimard.

-, 2009, "Parcours politique d'une apparition. Le cas de Lourdes ", Archives de sciences sociales des religions, $\mathrm{n}^{\circ} 145$, p. 109-128.

De Certeau Michel, 2005, La possession de Loudun, Paris, Gallimard.

Delooz Pierre, 1997, Les miracles : un défi pour la science?, Bruxelles, De Boeck/ Duculot.

Edelman Nicole, 2003, Les métamorphoses de l'hystérique. Du début du XIXe siècle à la Grande Guerre, Paris, La Découverte.

Foucault Michel, 1971, L'ordre du discours, Paris, Gallimard.

-, 1999, Les anormaux, cours au Collège de France, 1974-1975, Paris, Seuil/Gallimard.

Freuler Léo, 1997, La crise de la philosophie au XIX ${ }^{e}$ siècle, Éditions J. Vrin, Paris.

-, 2005, Guérir, colloque à Lourdes, 25-26 novembre 2004, Lourdes, NDL Éditions.

-, Octobre 1993, Guérisons et miracles, rapport du congrès international de Lourdes.

Guillaume Pierre, 1990, Médecins, Église et foi, XIXe-XXe siècles, Paris, Éditions Aubier.

Guillemain Hervé, 2003, "Les début de la médecine catholique en France. La société médicale Saint-Luc, Saint-Côme et Saint-Damien (1884-1914) ", Revue d'histoire du XIX $X^{e}$ siècle, 26-27, p. 227-258.

Gusdorf Georges, 1969, Les sciences humaines et la pensée occidentale III : la Révolution Galiléenne, t. 1, Paris, Payot.

HARRIS Ruth, 2001, Lourdes [1999, Lourdes. Body and Spirit in The Secular Age], Paris, J.-C. Lattès.

LALOUETTE Jacqueline, 1989, "Science et foi dans l'idéologie libre penseuse (1866-1914) » in Christianisme et Science, études réunies par l'Association française d'Histoire religieuse contemporaine, Paris, J. Vrin, p. 21-54.

Laurentin René, 1958, Lourdes. Documents authentiques, t. 3, Paris, P. Lethielleux.

-, 1959, Lourdes. Documents authentiques, t. 5, Paris, P. Lethielleux.

LE BEC Édouard, 2004, Miracles de Lourdes. Les preuves médicales, Clamecy, Presses de Valmy.

Lenoble Robert, 1969, Histoire de l'idée de nature, Paris, Albin Michel.

LÉONARD Jacques, 1978, La France médicale. Médecins et malades au XIXe siècle, Paris, Gallimard.

-, 1981, La médecine entre les pouvoirs et les savoirs, Paris, Éditions Aubier.

MangIaPAN Théodore, 1994, Les guérisons de Lourdes. Étude historique et critique depuis l'origine à nos jours, Lourdes, œuvre de la Grotte.

Mead Georges Herbert, 2006, L'esprit, le soi et la société [1934, Mind, Self and Society from the Standpoint of a Social Behaviorist], Paris, Presses universitaires de France.

Nietzsche Friedrich, 1993, Euvres, Paris, Robert Laffont.

NoIray Jacques, "préface » in Zola, E., Lourdes, Paris, Gallimard, 1995, p. 7-27.

OGorzelec Laetitia, 2010, «De la foule à la procession. La mise en place d'une stratégie de contrôle social à Lourdes ", ethnographiques.org, n 21, en ligne.

-, 2011, «Le miracle et l'enquête ", Sociologie, vol. 3, p. 243-258. 
Poulat Émile, 1996, Histoire, dogme et critique dans la crise moderniste, Paris, Albin Michel.

Quéré Louis, 2004, «Il faut sauver les phénomènes! Mais comment ? », Espaces Temps, volume $84, n^{\circ} 84-86$, p. 24-37.

Renan Ernest, 1890, L'avenir de la science, Paris, Calmann Lévy.

Rodé François, 1965, Le miracle dans la controverse moderniste, Paris, Beauchesne.

SAINTYVES Pierre, 1909, Le discernement du miracle, Paris, Librairie critique Émile Nourry.

Salmon Michel, 1959, Les guérisons extraordinaires de Lourdes.

STRAuSs Anselm, 1992, La trame de la négociation, Paris, L'Harmattan.

VALot Thérèse et Guy, 1956, Lourdes et l'illusion, Paris, Librairie Maloine.

Weber Max, 1971, Économie et société, t. 1, Paris, Plon.

Woodward Kenneth, 1992, Comment l'Église fait les saints [1990, Making Saints], Paris, Grasset.

Zola Émile, 1894, Lourdes, Paris, G. Charpentier et É. Fasquelle éditeurs.

-, 2004, Le Docteur Pascal, Paris, Le livre de poche classique.

\section{L'évolution de l'expertise médicale des guérisons de Lourdes au regard de la " conversation de gestes"}

Par-delà une confrontation statique entre rationalistes et médecins catholiques, cet article propose de revenir sur la "conversation de gestes " qui s'instaure, dès la fin $d u X_{X} X^{e}$ siècle, entre les médecins du Bureau médical du sanctuaire de Lourdes, chargés d'expertiser les guérisons déclarées "miraculeuses ", et leurs détracteurs : positivistes, médecins athées ou libres penseurs... En suivant les réajustements qu'ils mettent en cuvre dans la phase médicale de la procédure de reconnaissance des miracles à Lourdes, notre analyse montre que les médecins du sanctuaire s'efforcent de se maintenir "dans le vrai " du discours médical de leur époque afin de continuer à établir, au terme de leurs enquêtes, les certitudes que l'Église attend d'eux.

Mots-Clés : expertise médicale, conversation de gestes, Lourdes, miracle, hystérie.

\section{The evolution of the medical expertise of Lourdes' cures relatively to the "conversation de gestes"}

More than the static confrontation between rationalist and catholic doctors, this article suggests returning on the "conversation" (in the pragmatic sense of the term) established, at the end of the $19^{\text {th }}$ century, between the doctors of the Medical Office of the sanctuary of Lourdes, asked to evaluate the so called "supernatural" remissions, and their detractors: positivists, atheistic doctors or free thinkers... Following the adjustments which they set in the medical phase of the procedure of recognizing Lourdes miracles, we show that the doctors of the sanctuary try hard to remain "truthful" following the medical discourse of the time to keep on setting, at the end of their inquiries, the certainties which the Church expects from them.

Key words: medical expertise, conversation by gestures, Lourdes, miracle, hysteria. 


\section{La evolución de la expertise médica de las sanaciones de Lourdes en relación con la "conversación de gestos"}

Más allá de una confrontación estática entre racionalistas y médicos católicos, este artículo propone volver sobre la "conversación de gestos" que se instaura, desde fines del siglo XIX, entre los médicos del Centro Médico del Santuario de Lourdes, encargados de investigar las sanaciones declaradas "milagrosas", y sus detractores: positivistas, médicos ateos o librepensadores... Siguiendo los reajustes que se ponen en obra en la fase médica del procedimiento de reconocimiento de los milagros en Lourdes, mostramos que los médicos del santuario se esfuerzan por mantenerse "en la verdad" del discurso médico de su época para poder seguir estableciendo, al final de sus investigaciones, lo que la Iglesia espera de ellos.

Palabras clave: expertise médica, conversación de gestos, Lourdes, milagro, histeria. 
